\title{
MULTIPLE FEATURES BASED FINGERPRINT IDENTIFICATION SYSTEM
}

\author{
Sumitha.S.M ${ }^{1}$, A.N.Mukunda Rao ${ }^{2}$ \\ ${ }^{1}$ PG Scholar, Dept of Electronics and Communication, Siddaganga Institute of Technology, Karnataka, India \\ ${ }^{2}$ Associate Professor, Dept of Electronics and Communication, Siddaganga Institute of Technology, Karnataka, India
}

\begin{abstract}
Security has become major issue now a day. In order to prevent unauthorized access of confidential data there is a need for accurate and reliable personal identification system. So, biometric based identification system is one of the best solutions. Fingerprint based system is one of oldest biometric identification systems. It is used in many commercial and security applications. Even with advent of technology in fingerprint identification system, the accurate extraction and matching of features from a fingerprint image is a challenging task. The task is much more challenging when fingerprint is affected by non-linear deformations such as rotation and translation. In this paper, fingerprint identification system using improved feature vector based algorithm is presented. In the algorithm Gabor filter is implemented to enhance the fingerprint image. The salient features minutiae (ridge endings) and reference point are extracted from the image. The Euclidian distances between reference point and each minutiae point are calculated and are arranged in ascending order. These are stored in database as feature vectors. The fingerprint matching is done based on the similarity rate between the feature vector of input fingerprint and the feature vectors stored in the database. Algorithms are implemented using Visual Studio 2010 in C++ language using Open CV libraries and tested on the fingerprint database created in the laboratory.
\end{abstract}

Key Words: Fingerprint, Minutiae, Reference point, Euclidian distance, Similarity rate, Identification $* * *$

\section{INTRODUCTION}

Biometric systems are automated systems used to identify a person or verify his/her identity based on behavioral or physiological characteristics. The term biometric is derived from the Greek words, bio which means life and metric means to measure. Biometric systems have the ability to correctly recognize whether it is an authorized person or an imposter. The biometric traits include fingerprints, face, hand geometry, iris scans, ear, speech, gait, signature, key stroke dynamics and odor. Among all the biometrics the fingerprint based identification is one of the important and reliable techniques. Due to high level of uniqueness and permanence of fingerprint, the system has picked up enormous popularity. The solid state sensors are easily available at lower cost. So that it can be embedded in various devices that require user authentication. Fingerprint authentication is having higher user acceptance compared to other biometrics. Moreover, fingerprints cannot be forgotten and it is very difficult to steal and reproduce them. An example for a fingerprint captured using optical fingerprint sensor is shown in Fig 1.

In the finger tip, a ridge is the raised portion and the gap between two consecutive ridges is termed as a valley. In the fingerprint images, the dark lines are the ridges and the brighter ones are valleys. This is shown in Fig 1. There are five types of fingerprint. They are arch (Fig 2(a)), tented arch (Fig 2(b)), whorl (Fig 2(c)), right loop (Fig 2(d)) and left loop (Fig 2(e)).

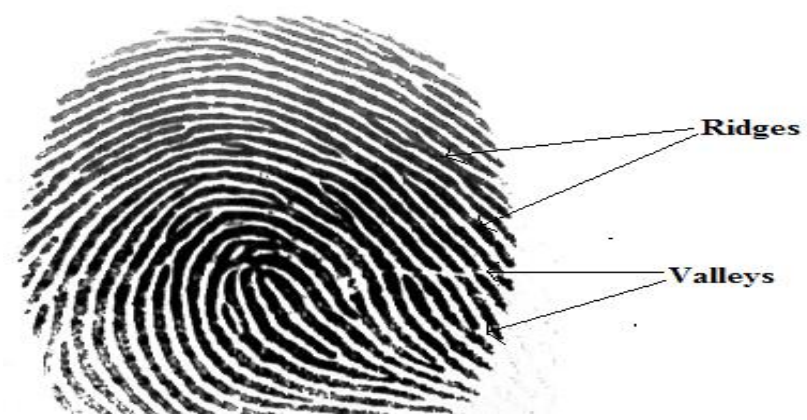

Fig -1: A fingerprint image captured using optical fingerprint sensor.

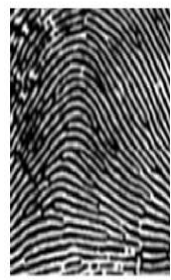

(a)

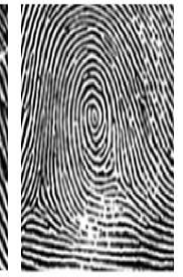

(c)

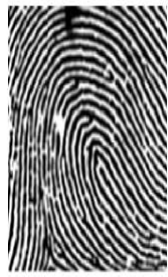

(d)

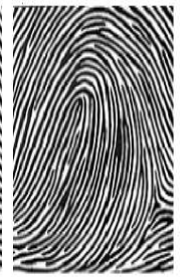

(e)
Fig -2: Types of fingerprint. (a)Arch, (b)Tented arch, (c)Whorl, (d)Right loop, (e)Left loop.

Fingerprint features are categorized into three levels. This is shown in Fig 3. Features in level-1 are ridge orientation field and singular points such as core and delta. These features give the macro details of the fingerprint such as flow of ridges and pattern type. The ridge orientation field is defined by local ridge orientation. Core point is a point at which ridge curvature is maximum and most it lies at center of the 
fingerprint image. Delta point is on a ridge at or in front of and nearest the center of the divergence of the type lines. Even though these features do not have uniqueness, they are useful for classification of fingerprint. Features in level-2 include minutiae points such as ridge bifurcations and ridge endings. These features would differentiate a person form others and it preserves individuality. Features in level-3 incorporate attributes in all dimension of a ridge such as ridge path deviation, shape, width, pores, breaks, incipient ridges, scars, edge contour and creases. These features also discriminate individual's fingerprint as they are permanent, immutable and unique.

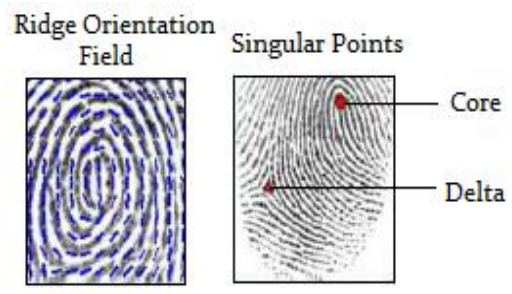

(a)

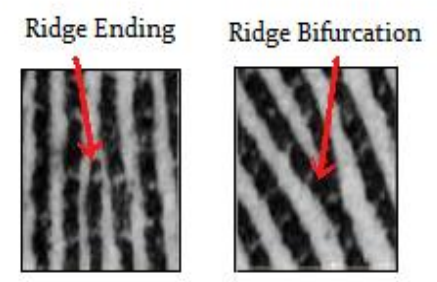

(b)
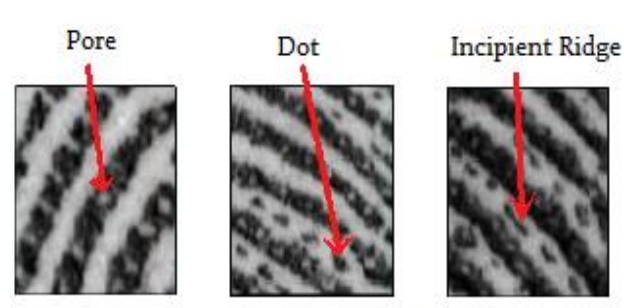

Ridge Edge

Protrusion

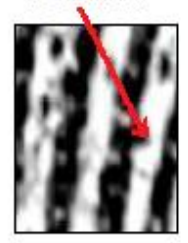

(c)

Fig -3: Fingerprint features. (a)Level-1 features (Ridge orientation field and singular points), (b)Level-2 features (Ridge ending and ridge bifurcation), (c)Level-3 features (pore, dot, incipient ridge and ridge edge protrusion).

Fingerprint recognition is a complex pattern recognition problem. Designing of an accurate algorithm that is able to extract and match the features of a fingerprint image in robust way is a challenging task. The real challenges in matching fingerprints are: (i)High displacements/rotation (ii)Distortion (iii)Different pressure and skin conditions (iv)Errors occur during extraction of feature may result in false or missing features. A general fingerprint identification system is shown in Fig 4. It operates in two phases.

\section{- $\quad$ Enrollment phase}

- Identification phase

Enrollment phase - In this phase fingerprint images are captured and it undergoes pre-processing stage. Then features are extracted and stored in database.

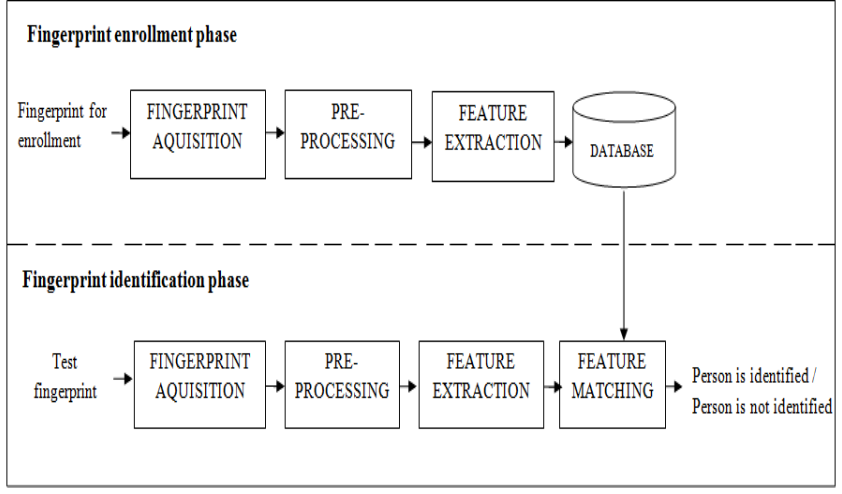

Fig -4: General fingerprint identification system.

Identification phase - In this phase first the test fingerprint is captured and its features are calculated. Then it is matched with the features of stored fingerprint images in the database. The matching score is obtained. If the matching score is above the predetermined threshold value, then the system outputs the positive decision (i.e., person is identified) otherwise it outputs the negative decision (i.e., person is not identified). Also it provides the identity of the person if the person is identified.

\section{RELATED WORK}

The most of the conventional fingerprint authentication system are based on minutiae features. One of such systems is proposed by Anil K. Jain [1]. In this approach minutiae matching are done in 2 stages. First is alignment stage and then matching stage. In alignment stage the input minutiae are aligned with minutiae in template by estimating the parameters such as rotation, scaling and translation between an input and a template in the database. In the matching stage the number of overlapping minutiae points and minutiae points differ by small distance are calculated. Based on the number of minutiae points matched the decision is taken. In this approach the problem of non linear deformation is overcome by alignment stage before minutiae matching. But there is false minutiae extraction in case of poor quality fingerprint. Ridge feature based matching is proposed by Anil K. Jain [2]. In this approach the feature vector that is FingerCode obtained by applying bank of Gabor filter to the circular region around the core point of fingerprint. Matching is based on Euclidian distance between the FingerCodes of input and a template. The computational complexity is low. So, it works faster but it does not overcome the problem of non linear deformations.

Lot of work has been carried out for designing fingerprint recognition system using multiple features. It overcomes the constraints like non linear deformations and also it increases the performance of the system. One of such systems is proposed by Arun Ross [3]. In that, minutiae and texture features are considered. Texture features are obtained using set of 8 Gabor filters. Texture feature matching is based on Euclidian distance between the features of input and a template. Minutiae matching are based on number of overlapping minutiae points of input fingerprint and template. Fusion is done at score level using sum rule. 
Performance is better compared to the method based on minutiae alone.

Another approach in which orientation field and minutiae are considered as features. This method is proposed by Jinwei $\mathrm{Gu}$ [4]. The orientation field covers global and minutiae cover local information of the fingerprint. Combination model is used for orientation field matching. General Hough transform is used for minutiae matching. Fusion is done in decision level. This approach requires less storage and has high performance than Hybrid matcher [3]. Instead of orientation field as feature ridge density map is considered along with the minutiae. This method is proposed by Dingrui Wan [5]. Ridge density map is calculated by finding the inter ridge distance at each pixel. This ridge density map is modeled by polynomial approximation. Weighted Least Square (WLS) algorithm is used for polynomial approximation. Hough-transform based alignment is done in ridge density map matching. Fusion is at decision level using Neyman-pearson rule. This method performs better than the minutiae based method. As only model parameters are stored the memory required is less.

There is another method in which ridge features with minutiae is considered. This method is proposed by Heeseung Choi [6]. Minutiae extraction is done using cross number method. Ridge based coordinate system is considered to extract ridge feature vector which consists of ridge count, ridge length, ridge curvature direction and ridge type. By dynamic programming the matched ridge based coordinate system is detected. Using breath first search matched ridge features are detected. Matching is done for corresponding minutiae pairs of matched ridge feature vector to calculate minutiae matching score. Depending on the score, decision is taken. This approach overcomes problem of non-linear deformation as the ridge features are considered which remains same for any non-linear deformation.

There is another method in which core point and minutiae are considered. This method is proposed by Ala Balti [7]. Core point is detected using orientation at each pixel in fingerprint. Using crossing number method minutiae are extracted. Feature vector that consists the Euclidian distance between minutiae and core point is arranged in ascending order. Feature vector for ridge bifurcations and ridge ending is stored separately. Matching is based on similarity between input feature vector and feature vectors stored in database. Separate scores for ridge ending and ridge bifurcation are calculated. Score level fusion is done. In this method computational complexity is less and also it overcomes the problem of geometric rotation and translation that occur during acquisition of fingerprint image.

HOG (Histogram of Oriented Gradients) is a feature extracted from fingerprint. Minutiae and HOG can be used as features for fingerprint recognition. This approach is proposed by Gaurav Aggarwaltt [8]. Fingerprint is enhanced and minutiae are extracted. Fingerprint is divided into blocks. In the block if a minutia is present then HOG is calculated for sub regions in neighborhood of minutiae.
HOG gives textural information of fingerprint. Then histogram of all segments is combined together to form bag of HOG feature. Matching is based on calculating the correlation between bags of HOG features of input and the stored template.

There is other approach in which Level 3 features pores and ridges are considered. This method is proposed by Anil K. Jain [9]. Pores and ridges are extracted using Gabor filter and wavelet transform. By linear combination of Gabor filtered image and wavelet response and by applying threshold pore features are extracted. Ridge contour enhancement is done by linear subtraction of wavelet response and Gabor filtered image. Then it is binarized and thinned to get ridge feature. Here high resolution fingerprint image is required (1000ppi). Equal error rate is decreased when these features are combined with level 1 or level 2 features. Matching and fusion is hierarchical.

It can be observed that by considering multiple features the performance of the fingerprint recognition system is improved and it also overcome the constraints like non linear deformations which were faced by the system considering single feature. So, fingerprint identification system explained in this paper is based on two features. They are minutiae (ridge endings) and reference point (core point).

\section{PROPOSED APPROACH}

In fingerprint identification system process involved are fingerprint acquisition, pre-processing, feature extraction and feature matching.

\subsection{Pre-processing}

Fingerprint sensor is used to capture a digital image of the fingerprint pattern. The captured image is called as live scan. Fingerprint pattern of user can be captured using thermal, capacitive or optical fingerprint sensor. The fingerprint sensor used is optical fingerprint sensor Secugen Hamster Plus. The size of the fingerprint image captured is $260 \times 300$ pixels and the resolution is 500 DPI. Fingerprint image captured is in bitmap format (gray image). Fingerprint image captured contains noise which is not suitable to directly extract the features. So, it has to be enhanced. The steps involved in pre-processing are shown in Fig 5.

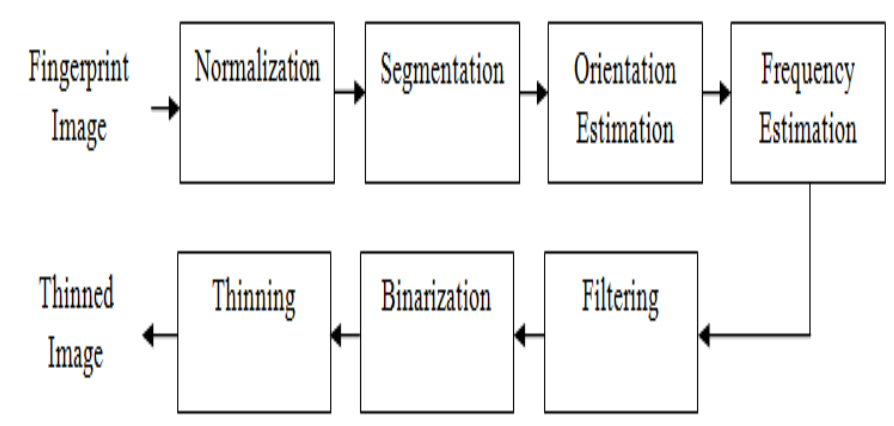

Fig -5: General fingerprint identification system. 


\subsubsection{Normalization}

The first step in pre-processing is normalization. Normalization is a process to decrease the variations in the gray level values along ridges and valleys [10]. After normalization the clarity of ridge and valley structures are not changed.

\subsubsection{Segmentation}

The process in which the foreground regions in the image are separated from the background regions is called segmentation. The area of interest is the foreground regions which contains clear fingerprint area where the ridges and valleys are present. There is no valid information of fingerprint present in the region outside the border of the fingerprint which is called as the background region. During minutiae extraction from these background regions noisy and false minutiae are extracted. So, segmentation is used to retain only the foreground region excluding background region, which helps in extraction of valid minutiae. The gray scale variance value is very low at the background regions and is very high at the foreground regions for the fingerprint image. Hence, segmentation can be performed using variance threshold method [11].

\subsubsection{Orientation estimation}

The local orientation of the ridges preset in the fingerprint is defined by the orientation field of a fingerprint image. When Gabor filter is used to enhance the fingerprint image there is requirement for orientation estimation. To compute the orientation image the least mean square estimation method is used [10].

\subsubsection{Frequency estimation}

The gray level values along ridges and valleys in the direction normal to the local ridge orientation forms a sinusoidal shaped wave, if there is no minutiae and singular points present in local neighborhood. Therefore another intrinsic property of a fingerprint image is local ridge frequency. The fingerprint image is divided into nonoverlapping blocks, then $\mathrm{x}$-signature values are calculated for each block and it forms sinusoidal-shaped wave. Using the distance between the consecutive peaks in $\mathrm{x}$-signature the frequency is calculated [10].

\subsubsection{Filtering}

After the ridge orientation and ridge frequency information has been obtained, using these parameters even-symmetric Gabor filter can be constructed. A Gabor filter is a two dimensional band pass filter consists of a sinusoidal plane wave of a specific orientation and frequency and it is modulated by a Gaussian envelope. Gabor filters have frequency-selective and orientation-selective properties hence it is used to enhance the ridges and valleys of fingerprint [10]. When the Gabor filter used is tuned perfectly it preserves the ridge structures and reduces noise.

\subsubsection{Binarization}

The process in which a gray level image is converted into a binary image is called binarization. By this contrast between the ridges and valleys in a fingerprint image is improved and it also helps in extraction of minutiae. A locally adaptive binarization method can be used. It is the process in which pixel with value more than the mean intensity value of selected block will be transformed to a pixel with value 1 . Otherwise it will be transformed to a pixel with value 0 .

\subsubsection{Thinning}

The process in which foreground pixels are successfully eroded until they are with width of one pixel is called thinning. The thinning operation is done using two subiterations. At each sub-iteration it starts with considering the neighborhood of each pixel in the binary image. A pixel should be deleted or retained is decided based on a particular set of criteria for pixel deletion. Until no more pixels can be deleted sub-iterations are continued. It also preserves the connectivity of ridges [12].

\subsection{Feature extraction}

After a fingerprint image has been enhanced, the next step is to extract the features from the enhanced image. The features establish the uniqueness of an individual's fingerprint. The features to be extracted are minutiae (ridge endings) and reference point. After the minutiae extraction, the false minutiae should be removed.

\subsubsection{Minutiae extraction}

Minutiae are the minute, precise and trivial details of the fingerprint image. These mainly include ridge endings and ridge bifurcations which are shown in Fig 6. The most commonly used method of minutiae extraction is the crossing number concept [13]. In this method, the thinned image is used where the ridge pattern is eight connected. The minutiae are extracted by scanning the local neighborhood of each ridge pixel in the eight-neighborhood. Using the properties of the crossing number the ridge pixel can then be classified as a ridge ending, bifurcation or nonminutiae point. The crossing number $C N$ for a ridge pixel $P$ is given by equation 1 ,

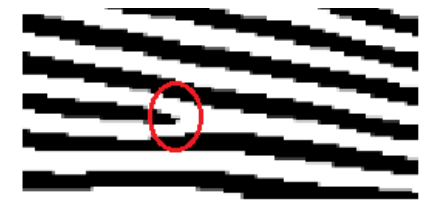

(a)

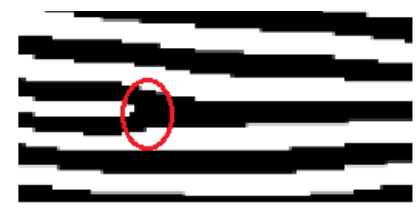

(b)
Fig -6: Minutiae. (a)Ridge ending, (b)Ridge bifurcation (Pixels with value 0 is considered as ridge)

\begin{tabular}{|c|c|c|}
\hline$P_{4}$ & $P_{3}$ & $P_{2}$ \\
\hline$P_{5}$ & $P_{1}$ & $P_{1}$ \\
\hline$P_{6}$ & $P_{7}$ & $P_{8}$ \\
\hline
\end{tabular}

Fig -7: Order of scan around pixel $P$ 


$$
C N=\frac{\sum_{i=1}^{\mathrm{E}}\left\|P_{i}-P_{i+1}^{i}\right\|}{2} \ldots \ldots \ldots(1)
$$

Where, $P_{\mathrm{i}}$ is the value of pixel in the neighborhood of $P$.

The eight neighboring pixels of a pixel $P$ is scanned in an order is shown in Fig 7. After obtaining the crossing number $C N$ using equation 1 , minutiae are marked. If $C N=1$ then pixel $P$ is ridge ending point and if $C N=3$ then pixel $P$ is ridge bifurcation point.

The fingerprint image is not totally healed by pre-processing stage. Though the scanned fingerprint image gets better clarity through pre-processing stage, each of the stages in pre-processing introduce small amount of errors that leads to extraction of false minutiae. Therefore removal of false minutiae is necessary in an effective fingerprint identification system.

To remove false minutiae following steps are carried out at each minutia such as ridge ending [14]:

- With the size of $W_{1} \times W_{1}$ an image $L_{1}$ is created and initialized with value 0 . A pixel located in $W_{1} \times W_{1}$ neighborhood of minutia in thinned image is corresponded by each pixel of $L_{1}$. Where the center of the image $L_{1}$ is positioned at minutia.

- The center pixel of $L_{1}$ is Labeled with -1 (Fig 8(a)). In the thinned image corresponding minutiae point is indicated by this pixel.

- In an image $L_{1}$ all the pixels which are corresponding to the pixels that are connected to the ridge ending is labeled with 1 (Fig 8(b)).

- At an image $L_{1}$ border, scanning is done in clockwise direction. The number of transitions from 0 to $1\left(T_{01}\right)$ are counted (Fig 8(c)).

- When $T_{01}=1$, the minutia is considered as valid ridge ending.

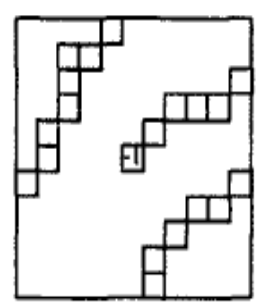

(a)

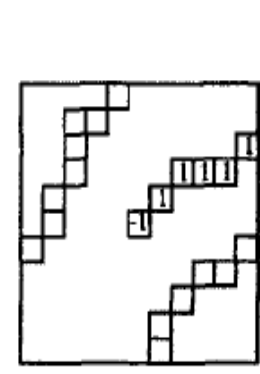

(b)

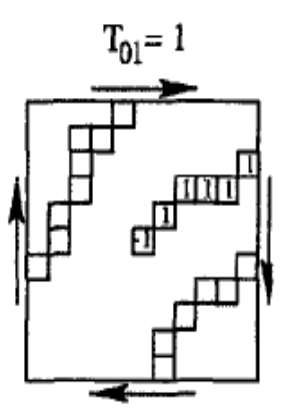

(c)
Fig -8: Steps to remove false ridge ending

Around each candidate minutia the neighborhood of dimension $W_{1}$ is analyzed. The dimension $W_{1}$ is chosen larger than two times the average inter-ridge distance and it should not be too large which would result in retaining of some false minutiae.
There exist false minutiae at boundary of fingerprint image even after false minutiae are removed. Some of pixels in border of fingerprint is marked as ridge ending but they are not actual ridge ending. These false minutiae are removed using plus rule [15]. The pixels around each minutia are scanned with a pattern of plus sign. The scanning is done to find presence of white pixels (i.e. ' 1 ' value) across these lines. If no white pixel is detected, in any one of the lines, the minutia is considered as boundary minutiae and it is eliminated.

\subsubsection{Reference point location}

The core point is the most commonly used reference point [16]. The core point of a fingerprint is a point at which the concave ridges have maximum curvature. This point occurs around the central portion of the finger tip. Therefore, in captured fingerprint image reference point lies somewhere around the central part of the image. This point is found using Poincare index method [10]. This method is applied after orientation field of a fingerprint image is estimated. The estimation of orientation field is done in pre-processing steps.

Let $w$ be the size of block considered for orientation estimation. Let $\theta(p, q)$ be the local orientation estimated at the block centered at pixel $(p, q)$ and $0 \leq \theta(p, q)<2 \pi$. The Poincare index is defined for each position $(p, q)$ at which local orientation is estimated and computed by considering local orientation of neighboring blocks. The 8 elements contiguous to the target position are considered. For a position $(p, q)$, let

$$
\begin{aligned}
& \left(p_{0}, q_{0}\right)=(p, q+w), \\
& \left(p_{1}, q_{1}\right)=(p+w, q+w), \\
& \left(p_{2}, q_{2}\right)=(p+w, q) . \\
& \left(p_{2}, q_{7}\right)=(p+w, q-w)_{x} \\
& \left(p_{4}, q_{4}\right)=(p, q-w), \\
& \left(p_{5}, q_{5}\right)=(p-w, q-w)_{x} \\
& \left(p_{6}, q_{6}\right)=(p-w, q) . \\
& \left(p_{7}, q_{7}\right)=(p-w, q+w) .
\end{aligned}
$$

Let

$$
\delta_{k}(p, q)=\theta\left(p_{k+1}, q_{k+1}\right)-\theta\left(p_{k}, q_{k}\right)
$$

For $0 \leq k \leq 6$ and $\delta_{7}=\theta\left(p_{0}, q_{0}\right)-\theta\left(p_{7}, q_{7}\right)$. Then, the Poincare index of an element $(p, q)$ is defined to be

$$
P(p, q)=\frac{1}{2 \pi} \sum_{k=0}^{7} \Delta_{k}(p, q) \ldots \ldots \ldots(
$$

Where,

$$
\Delta_{k}(p, q)=\left\{\begin{array}{cc}
\delta_{k}(p, q) \quad \text { if }\left|\delta_{k}(p, q)\right|<\frac{\pi}{2} \\
\pi+\delta_{k}(p, q) \text { if } \delta_{k}(p, q) \leq-\frac{\pi}{2} \ldots \ldots \ldots(4) \\
\pi-\delta_{k}(p, q) \quad \text { otherwise }
\end{array}\right.
$$


The Poincare index has the value $-1 / 2,0,1 / 2$, or 1 . A core point and a delta point are expected to occur at the position of which Poincare index is $1 / 2$ and $-1 / 2$, respectively. The point is invariant for rotations. An example for fingerprint image with reference point located is shown in Fig 9.

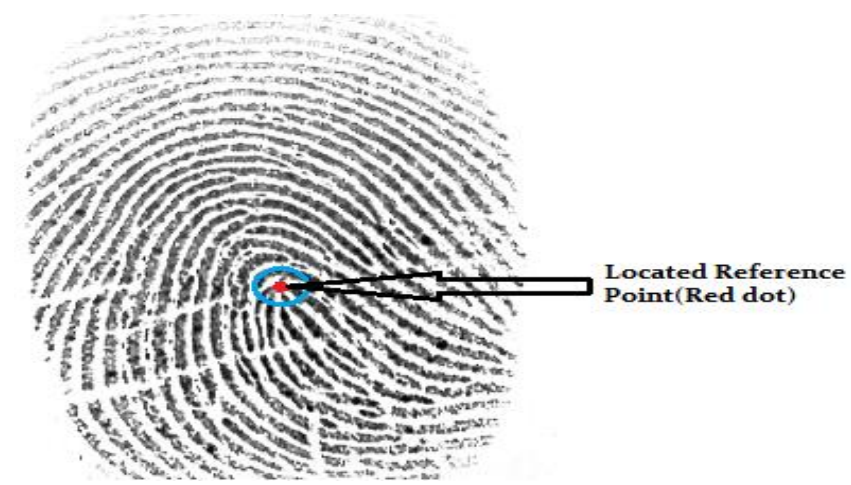

Fig -9: Reference point of fingerprint.

\subsubsection{Feature vector calculation}

The steps involved in calculating feature vector are:

- Reference point is detected.

- Minutiae points (ridge endings) are detected.

- For $j=1$ until $N_{\mathrm{p}}$, The Euclidian Distance between the reference point and minutiae points are computed using the formula

$$
E D(j)=\sqrt{\left(x_{c}-x_{M}(j)\right)^{2}+\left(y_{c}-y_{M}(j)\right)^{2}}
$$

Where, $N_{p}$ is the number of minutiae points. $x_{c}$ and $y_{c}$ are the spatial coordinates with $x$ and $y$ pixels for the reference point, $x_{M}$ and $Y_{M}$ are the spatial coordinates with $x$ and $y$ pixels for minutiae points.

- The Euclidian Distance between the reference point and deduced minutiae points are sorted in ascending order to get Euclidian distance vector.

- The Euclidian distance vector is stored in the database.

\subsection{Feature matching}

Fingerprint matching is based on similarity rate between the feature vector of input fingerprint and feature vectors stored in database. The Similarity rate $S R(\%)$ is given by

$S R(\%)=100-\left[\frac{\sum_{i=1}^{N_{D}} \sqrt{\left(E D_{R}(i)-E D_{T}(i)\right)^{2}}}{N_{p}}\right] \ldots \ldots$

Where $E D_{R}(i)$ the reference Euclidian distance vector is saved on the database and $E D_{T}(i)$ is the Euclidian distance vector of test fingerprint. $N_{p}$ is the length of the large feature vector. Zero padding is done for the small feature vector to make length equal to $N_{p}$.

These features are invariant for rotation and translation. The length of feature vectors and variation in Euclidian distance value along vector of particular fingerprint is similar and differ for samples of different fingerprint. Hence, similarity rate is high for samples of same person's fingerprint images. Then the decision $\mathrm{D}$ is taken according to

$$
D=\left\{\begin{array}{rr}
D_{p} & \text { if } 5 R(\%)=T \\
D_{n} & \text { otherwise }
\end{array} \ldots \ldots \ldots\right. \text { (7) }
$$

Where $D_{p}$ is the positive decision (valid user) and $D_{n}$ is negative decision (invalid user) and $T$ is the threshold value. For the given input fingerprint, if the decision obtained is positive then identity of the person is obtained by the class of feature vectors stored in database with which maximum similarity rate is obtained.

\section{EXPERIMENTAL RESULTS}

A fingerprint image database is created in the lab by taking right hand, fore-fingerprint images from 10 persons. From each person 15 fingerprint image samples are collected. From different persons other than those 10 members 50 fingerprints are randomly collected to calculate false acceptance of the fingerprint identification system. So a total of 200 fingerprint images are available in the database. The scanned fingerprint images are of bit map format with resolution 500dpi and the size of fingerprint captured is 260 $\times 300$ pixels. Among the 15 samples obtained from each person, Euclidian distance vectors are stored in database for 10 samples and remaining 5 samples are used for testing purpose.
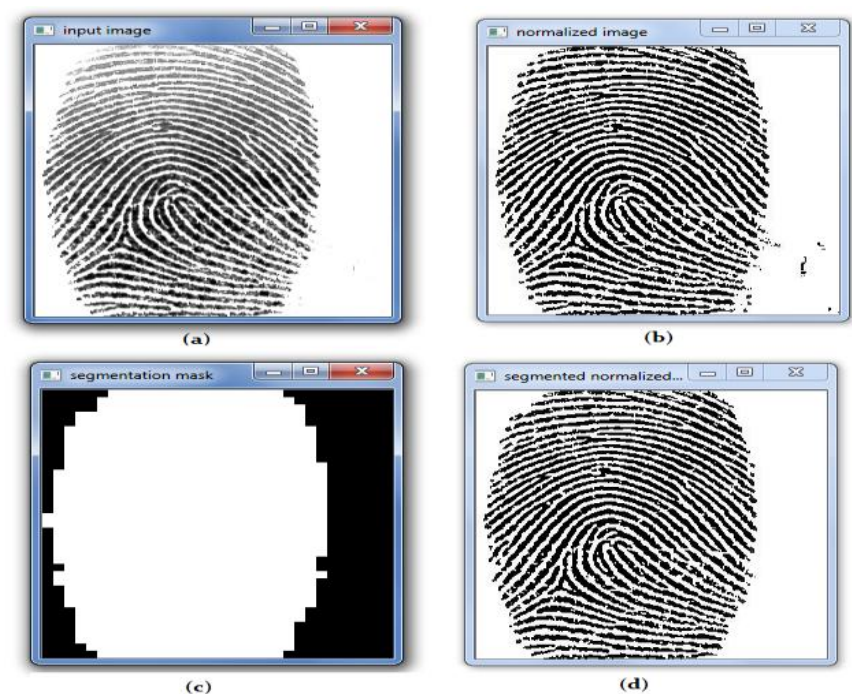

Fig -10: (a) Input image, (b) Normalized image, (c) Segmentation mask, (d) Segmented normalized image

Input fingerprint image is shown in Fig 10(a). Results obtained at preprocessing steps and feature extraction is shown in Fig 10 and Fig 11. Fingerprint image after normalization is shown in Fig 10(b). Segmentation mask is shown in Fig 10(c). Foreground regions are indicated by white and background region is indicated by black. Segmented normalized image is shown in Fig 10(d). Enhanced image obtained after Gabor filtering is shown in Fig 11(a). Inverse binarized image is shown in Fig 11(b). Thinned image obtained using Guo-Hall's thinning 
algorithm is shown in Fig 11(c). Minutiae are marked using cross number method is shown in Fig 11(d). Red dots indicate ridge bifurcation and green dots indicate ridge ending. False minutiae are removed at boundary of fingerprint using plus rule is shown in Fig 11(e). Connected component based method is used to remove false minutiae. The fingerprint image with only valid minutiae marked is shown in Fig 11(f). Core point of fingerprint marked is shown in Fig 11(g). Blue dot indicates core point.
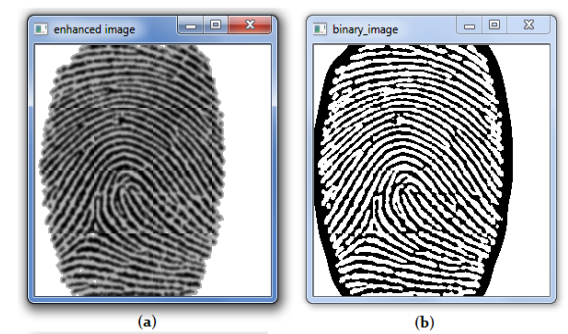

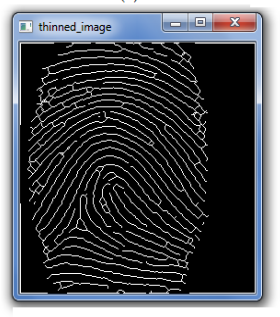

(c)

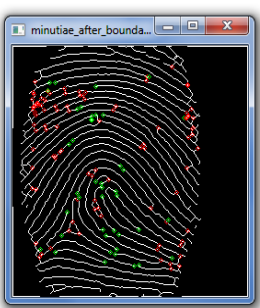

(e)

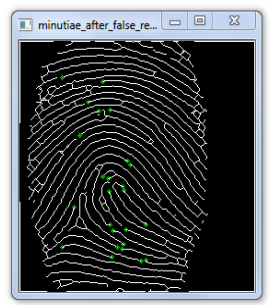

(f)

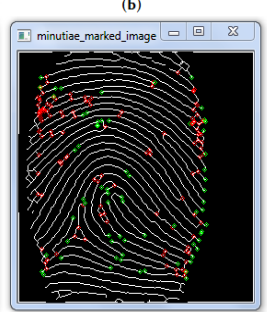

(d)

Fig - 11: (a) Enhanced image, (b) Inverse binarized image,

(c) Thinned image, (d) Minutiae marked image, (e) Minutiae after boundary elimination, (f) Valid minutiae after removing false minutiae, $(\mathrm{g})$ Core point of fingerprint

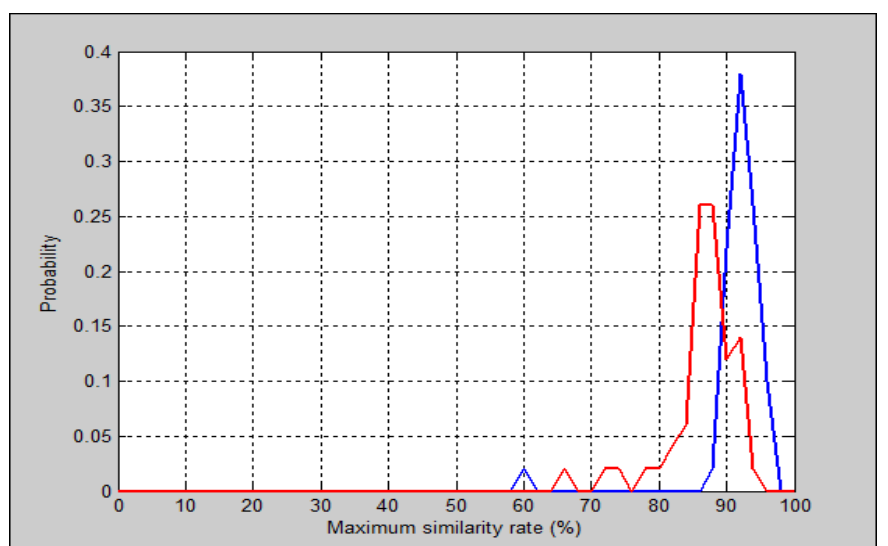

Chart -1: Probability distribution curves for authorized and unauthorized users fingerprints

At the matching stage for a given test fingerprint's feature vector, the similarity rate with each feature vector stored in database is calculated. So, maximum similarity rate for each fingerprint in set of test fingerprints of authorized users and in set of fingerprints of unauthorized users are calculated. The plot of maximum similarity rate v/s probability of occurrence of the maximum similarity rate in particular set of fingerprints is shown in Chart -1 .

The distribution curve with blue color indicates for fingerprints of authorized users and red color indicates for fingerprints of unauthorized users. For different threshold value the false acceptance rate and false rejection rates are listed in Table -1. Using these values the plot of FAR-FRR v/s threshold is shown in Chart -2 . It can be seen that the false acceptance rate decreases with increase in threshold but false rejection rate increases with increase in threshold. Equal error rate obtained for the fingerprint identification system is $19 \%$. From this plot the threshold value can be decided. At the threshold value of 90.5, the FAR and FRR values are optimum.

Table -1: False acceptance and false rejection rates with different threshold value for the database created in laboratory

\begin{tabular}{|l|l|l|}
\hline Threshold $(\%)$ & $\begin{array}{l}\text { False acceptance } \\
\text { rate }\end{array}$ & $\begin{array}{l}\text { False rejection } \\
\text { rate }\end{array}$ \\
\hline 85 & 80 & 2 \\
\hline 86 & 70 & 2 \\
\hline 87 & 54 & 2 \\
\hline 88 & 40 & 4 \\
\hline 89 & 28 & 4 \\
\hline 90 & 24 & 8 \\
\hline 91 & 16 & 26 \\
\hline 92 & 6 & 48 \\
\hline 93 & 2 & 64 \\
\hline 94 & 0 & 82 \\
\hline 95 & 0 & 90 \\
\hline
\end{tabular}

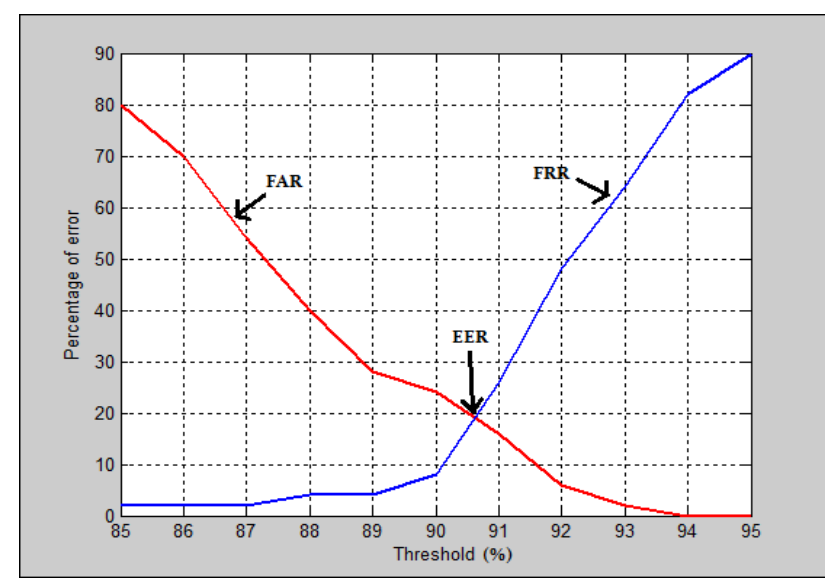

Chart -2: FAR-FRR curves of fingerprint identification system

Performance of fingerprint identification system is tabulated in Table -2 for threshold 90.5. The accuracy of the system is given by

(\%) Accur acy $=\frac{\text { No. of correct acceptance }+ \text { No. of correct rejection }}{\text { Total no. of test fingerprints }} \times 100$

$$
=\frac{46+38}{100} \times 100=84 \%
$$


Identification results of fingerprint identification system are tabulated in Table -3 for threshold 90.5 . The accuracy of the system is given by

$$
\begin{aligned}
(\%) \text { Accuracy } & =\frac{\text { No. of correct identifications }}{\text { Total no. of authorized test fingerprints }} \times 100 \\
& =\frac{99}{50} \times 100=78 \%
\end{aligned}
$$

Table -2: Performance of fingerprint identification system

\begin{tabular}{|l|l|l|}
\hline \multicolumn{1}{|c|}{ Actual } & $\begin{array}{l}\text { Authorized } \\
\text { user }\end{array}$ & $\begin{array}{l}\text { Unauthorized } \\
\text { user }\end{array}$ \\
\hline Authorized user & 46 & 4 \\
\hline Unauthorized user & 12 & 38 \\
\hline
\end{tabular}

Table -3: Identification results of fingerprint identification system for test fingerprint samples

N: Not identified (Rejected)

\begin{tabular}{|l|l|l|l|l|l|}
\hline $\begin{array}{l}\text { Authorize } \\
\text { d users } \\
\text { Samples }\end{array}$ & $\begin{array}{l}\text { Sampl } \\
\text { e } \\
1\end{array}$ & $\begin{array}{l}\text { Sampl } \\
\text { e 2 }\end{array}$ & $\begin{array}{l}\text { Sampl } \\
\text { e 3 }\end{array}$ & $\begin{array}{l}\text { Sampl } \\
\text { e }\end{array}$ & $\begin{array}{l}\text { Sampl } \\
\text { e 5 }\end{array}$ \\
\hline Person 1 & 1 & 1 & N & N & 1 \\
\hline Person 2 & 2 & 2 & 2 & 2 & 2 \\
\hline Person 3 & 3 & 3 & 3 & 3 & 1 \\
\hline Person 4 & 4 & 4 & 10 & 4 & 4 \\
\hline Person 5 & N & 5 & 5 & 5 & 5 \\
\hline Person 6 & 6 & 6 & 6 & 6 & 6 \\
\hline Person 7 & 4 & 7 & 9 & 7 & 7 \\
\hline Person 8 & 8 & 8 & 8 & 4 & N \\
\hline Person 9 & 9 & 9 & 4 & 9 & 9 \\
\hline Person 10 & 10 & 10 & 9 & 10 & 10 \\
\hline
\end{tabular}

\section{CONCLUSIONS AND FUTURE WORK}

The proposed method uses level 1 and level 2 fingerprint features to identify a person. A Euclidian distance vector is extracted from each fingerprint. The feature vector requires only few bytes for storage and hence saves memory. Matching stage involves only calculation of similarity rates and comparing then with threshold T. Hence, there is no complex computation. This proposed method overcomes the problem of geometric rotation and translations.

The time taken for fingerprint identification is 35 seconds on personal computer with Intel Core 2 Duo processor with speed $2.2 \mathrm{GHz}$ and the software used is Microsoft Visual Studio 2010 interfaced with Open CV 2.4.8 library. So, there is scope for improvement in order to obtain satisfactorily fast fingerprint identification system. The error in estimation of local ridge orientation and ridge frequency cause block artifacts in the enhanced image. This leads to occurrence of many false minutiae. So, accurate estimation of local ridge orientation and ridge frequency would improve the fingerprint enhancement and occurrence of false minutiae would be less. Along with the Euclidian distance between minutiae and reference point, orientation of the line joining minutiae and reference point can also be considered as the feature vector and that would increase the performance of the fingerprint identification system.

\section{REFERENCES}

[1]. Lin Hong, Anil Jain, Sharath Pankanti and Ruud Bolle, "Identity Authentication Using Fingerprints", Proceedings of the IEEE, Vol. 85, No. 9, pp. 13651388, 1997.

[2]. Anil K. Jain, Salil Prabhakar, Lin Hong and Sharath Pankanti, "Filterbank-Based Fingerprint Matching", IEEE Transactions on Image Processing, Vol. 9, No. 5, May 2000.

[3]. Anil k. Jain, Arun Ross and Salil Prabhakar, "Fingerprint Matching Using Minutiae And Texture Features", Appeared in Proceedings Of International Conference on Image Processing (ICIP), pp. 282-285, Thessaloniki, Greece, Oct 7-10, 2001.

[4]. Jinwei Gu, Jie Zhou and Chunyu Yang, "Fingerprint Recognition by Combining Global Structures and Local Cues", IEEE Transactions on Image Processing, Vol. 15, No. 7, July 2006.

[5]. Dingrui Wan and Jie Zhou, "Fingerprint Recognition Using Model-Based Density Map”, IEEE Transactions on Image Processing, Vol. 15, No. 6, June 2006.

[6]. Heeseung Choi, Kyoungtaek Choi and Jaihie Kim, "Fingerprint Matching Incorporating Ridge Features with Minutiae", IEEE Transactions on Information Forensics and Security, Vol. 6, No. 2, June 2011.

[7]. Ala Balti, Mounir Sayadi and Farhat Fnaiech, "Improved features for fingerprint identification", Appeared in Proceedings Of IEEE conference on Electrotechnical, pp 878-883, Yasmine Hammamet, Mar 25-28, 2012.

[8]. Gaurav Aggarwaltt, Nalini K. Rathat, Tsai-Yang Jeat and Ruud M. Bollet, "Gradient based Textural Characterization of Fingerprints", Appeared in Proceedings Of IEEE conference on Biometrics: Theory, Applications and Systems, pp 1-5, Arlington, VA, Sept.29-Oct.1, 2008.

[9]. Anil K. Jain, Yi Chen and Meltem Demirkus, "Pores and Ridges: High-Resolution Fingerprint Matching Using Level 3 Features", IEEE Transactions on Pattern Analysis And Machine Intelligence, Vol. 29, No. 1, January 2007.

[10]. Lin Hong, Yifei Wan and Anil Jain, "Fingerprint Image Enhancement: Algorithm and Performance Evaluation", IEEE Transactions on Pattern Analysis And Machine Intelligence, Vol. 20, No. 8, August 1998.

[11]. Ishmael S. Msiza, Mmamolatelo E. Mathekga, Fulufhelo V. Nelwamondo and Tshilidzi Marwala2, "Fingerprint Segmentation: An Investigation Of Various Techniques And A Parameter Study Of A 
Variance-Based Method", International Journal of Innovative Computing, Information and Control, Volume 7, Number 9, September 2011.

[12]. Z. Guo and R. Hall, "Parallel thinning with twosubiteration algorithms", Communications of the ACM, Vol. 32, pp. 359-373, Mar 1989.

[13]. Manvjeet Kaur, Mukhwinder Singh, Akshay Girdhar and Parvinder S. Sandhu, "Fingerprint Verification System using Minutiae Extraction Technique", World Academy of Science, Engineering and Technology, Vol. 2, Oct 2008.

[14]. Marius Tico and Pauli Kuosmanen, "An Algorithm for Fingerprint Image Postprocessing”, IEEE Conference Record of the Thirty-Fourth Asilomar Conference on Signals, Systems and Computers, pp. 1735 - 1739, Vol. 2, Nov 2000.

[15]. Shahida Jabeen and Shoab A. Khan, "A Hybrid False Minutiae Removal Algorithm with Boundary Elimination", IEEE International Conference on System of Systems Engineering, pp. 1-6, June 2008.

[16]. Ali Ismail Awady and Kensuke Baba, "Singular Point Detection for Efficient Fingerprint Classification", International Journal on New Computer Architectures and Their Applications, 2012. 